\title{
Substituent contribution to the genotoxicity of benzophenone-type UV filters
}

\author{
Huimin Zhao a,b, Dongbin Wei ${ }^{\text {a,* }}$, Min $\mathrm{Li}^{\mathrm{a}}$, Yuguo Du ${ }^{\mathrm{a}}$ \\ a State Key Laboratory of Environmental Chemistry and Ecotoxicology, Research Center for Eco-Environmental Sciences, Chinese Academy of Sciences, \\ Beijing 100085, PR China \\ ${ }^{\mathrm{b}}$ School of Chemical and Environment Engineering, Wuyi University, Jiangmen, Guangdong Province 529020, PR China
}

\section{A R T I C L E I N F O}

\section{Article history:}

Received 3 April 2013

Received in revised form

22 May 2013

Accepted 28 May 2013

Available online 20 June 2013

Keywords:

SOS/umu assay

Genotoxicity

Benzophenone

UV filter

QSAR

\begin{abstract}
A B S T R A C T
Benzophenones (BPs) are widely used in UV filters, fragrance enhancers, and plastic additives. In this study, the genotoxicity of 14 BPs was tested using the SOS/umu assay, and the related substituent contribution was disclosed. The results of this study revealed that the major contributor to the genotoxicity of the BPs was the ortho,para-di-substitution, and the increasing hydroxy substitution on the benzene ring. In addition, the higher the dispersion of the substituent species on the two benzene rings, the lower the genotoxicity exhibited by the compound. Furthermore, 2 dimensional and 3 dimenional quantitative structure-activity relationships (2D- and 3D-QSAR) studies indicated that hydrogen-bond interactions and electrostatic effects were determinants for the genotoxicity of the BPs. The current results provide useful information for the assessment of the potential ecological risk and health effects of BP-type UV filters.
\end{abstract}

(c) 2013 Elsevier Inc. All rights reserved.

\section{Introduction}

UV-absorbing chemicals, such as UV filters, are widely used in sunscreens and cosmetics (Rodil and Moeder, 2008) and are added during the manufacturing process to insecticides, agricultural chemicals and pharmaceuticals (Roelandts et al., 1983; Tomson et al., 1981). A current survey showed that 26 organic UV filters were frequently used in personal care products (Rodil and Moeder, 2008). Therefore, the potential environmental impact of the increased use of sunscreens and cosmetics is of interest. UV filters applied to the skin may be directly washed off during swimming or bathing (Thomas et al., 2004). In addition, UV filters may be indirectly introduced into the environment through wastewater treatment plants as a result of treating the wash water from sunscreencontaminated towels and from sunscreen washed off during showers after dermal application. Although the daily consumption of UV filters is low, the environmental problem caused from their long-term input should not be neglected. Benzophenones (BPs) are important members of the UV filters family (Suzuki et al., 2005) and are natural components of plants, such as mango and Muscat grape, used as flavorings. Some benzodiazepine pharmaceuticals and the corresponding human metabolites could form benzophenone

\footnotetext{
* Correspondence to: 18 Shuangqing Road, Haidian District, Beijing 100085 , PR China. Fax: +861062923563.

E-mail addresses: wyuchemys10zhm@126.com (H. Zhao), weidb@rcees.ac.cn (D. Wei), bkdlimin@126.com (M. Li), duyuguo@rcees.ac.cn (Y. Du)
}

derivatives during the aqueous photo-transformation process (West and Rowland, 2012). Jeon et al. (2008) found that, in rats, BPs could be converted to benzohydrol and 4-hydroxy-BP, and 2-hydroxy-4methoxy-BP could be converted to 2,4-dihydroxy-BP, 2,3,4-trihydroxy-BP, and 2,2'-dihydroxy-4-methoxy-BP. Detection of BP-type UV filters have been found within multiple environmental media and organisms (Balmer et al., 2005; Jeon et al., 2006; Loraine and Pettigrove, 2006; Negreira et al., 2011; Poiger et al., 2004). The concentrations of 2-hydroxy-4-methoxy-BP and 2-hydroxy-4-methoxy-5-sulfonic acid-BP in raw water and effluent were $1.5-19 \mu \mathrm{g} / \mathrm{L}$ and $0.01-2.7 \mu \mathrm{g} / \mathrm{L}$, respectively (Vosough and Mojdehi., 2011; Li et al., 2007; Kupper et al., 2006; Liu et al., 2012; Fent et al., 2010). In addition, the accumulated concentration of 2-hydroxy-4-methoxy-BP in the Glatt River, Switzerland was up to $42 \mathrm{ng} / \mathrm{L}$ (Negreira et al., 2010). The average concentration of 5 BP-type UV filters was $0.38 \mathrm{ng} / \mathrm{g}$ in sediment samples from the Songhua River, China, (Zhang et al., 2011). Five BP-type UV filters (2-hydroxy-4-methoxy$\mathrm{BP}$, 4-hydroxy-BP, 2,4-dihydroxy-BP, 2,2'-dihydroxy-4-methoxy-BP and 2,2',4,4'-tetrahydroxy-BP) were detected within indoor dust from the U.S., Korea, Japan and China, with a median total concentration ranged from 78.3 to $612 \mathrm{ng} / \mathrm{g}$ (Wang et al., 2013). Urinary concentrations of BP-type UV filters in U.S. women were 6.1 $(<0.28-5900) \mu \mathrm{g} / \mathrm{L}$ for 2-hydroxy-4-methoxy-BP, $6.1 \quad(<0.082-$ 3200) $\mu \mathrm{g} / \mathrm{L}$ for 2,4-dihydroxy-BP, and $0.36(<0.082-22) \mu \mathrm{g} / \mathrm{L}$ for 4-hydroxy-BP (Kunisue et al., 2012).

Although the acute and subchronic systemic toxicity of these BP compounds after dermal application is considered to be rather low (Burdock et al., 1991; Dutta et al., 1993; Okereke et al., 1995), 
problems have been reported with photoallergic reactions in patients (Schauder and Ippen., 1997) and cytotoxic effects (Matsumoto et al., 2003). However, the potential ecological toxicity of BP-type UV filters that remain in the environment is of concern. Acute toxicity levels ( $48 \mathrm{~h} \mathrm{LC}_{50}$ ) of 2-hydroxy-4-methoxyBP and 2-hydroxy-4-methoxy-5-sulfonic acid-BP on Daphnia magna were determined as $1.9 \mathrm{mg} / \mathrm{L}$ and $50 \mathrm{mg} / \mathrm{L}$, respectively (Fent et al., 2010). The $48 \mathrm{~h} \mathrm{EC} \mathrm{EC}_{50}$ of 2,4-dihydroxy-BP on Acarti tonsa was $2.6 \mathrm{mg} / \mathrm{L}$ (Kusk et al., 2011), whereas the $72 \mathrm{~h} \mathrm{IC} \mathrm{IC}_{10}$ of 2-hydroxy-4-methoxy-BP on Demodesmus subspicatus was $0.56 \mathrm{mg} / \mathrm{L}$ (Sieratowicz et al., 2011). Currently, estrogenic activities (Schlumpf et al., 2004; Suzuki et al., 2005) and developmental effects (Weisbrod et al., 2007) of BP-type UV filters were focused on 4,4-dihydroxy-BP, 2,4-dihydroxy-BP and 2,2',4,4'-tetrahydroxy$\mathrm{BP}$, which exhibited estrogenic and additional hormonal activities in vitro (Kunz and Fent, 2006). Estrogenicity was displayed by 2-hydroxy-4-methoxy-BP in the MCF-7 breast cancer cell assay and in an immature rat uterotrophic assay. In addition, 2,4dihydroxy-BP and 2,2',4,4'-tetrahydroxy-BP displayed estrogenicity in fish (Kunz et al., 2006). Zucchi et al. (2011) confirmed that 2-hydroxy-4-methoxy-5-sulfonic acid-BP could interfere with the sex hormones and could alter the transcription of genes involved in hormonal pathways in zebrafish. Benzophenones were listed as "chemicals suspected of having endocrine-disrupting effects" by the World Wildlife Fund and the Japanese Ministry of Environment (MOE, 2000).

However, the genotoxicity effects of the BP-type UV filters have been ignored in the past decades, with the exception of a few sporadic reports (Abramsson-Zetterberg and Svensson, 2011; Nakajima et al., 2006; Jeon et al., 2007). For example, 2-hydroxy4-methoxy-BP, 2,4-dihydroxy-BP, 2-hydroxy-4-octyloxy-BP, and 2,2 -dihydroxy-4-methoxy-BP produced a positive or pseudopositive initiation activity in either the umu test or the Ames test. In particular, 2,4-dihydroxy-BP showed both initiation and promotion activities at a concentration of $10 \mu \mathrm{g} / \mathrm{mL}$ (Nakajima et al., 2006). Quantitative structure-activity relationships (QSARs) have been used extensively to screen and predict the fate and toxicity of organic chemicals released into the environment (Wei et al., 2002). Therefore, we detected the genotoxicity of BP-type UV filters (Fig. 1) using the SOS/umu assay and analyzed the genotoxicity-substituent relationships with these models for BP compounds having the same chemical skeleton. This study provides direct toxicity data and reveals the substituent contributions to the genotoxicity, which is beneficial for evaluating the potential ecological risk of the BP-type UV filters.

\section{Materials and methods}

\subsection{Reagents and chemicals}

The fourteen BP-type compounds ( $>97.5$ percent purity) were purchased from Sigma-Aldrich (St. Louis, MO, USA) (Table 1). These reagents were used for the genotoxicity tests directly, without further purification. Salts, including $\mathrm{Na}_{2} \mathrm{H}$ $\mathrm{PO}_{4} \cdot 12 \mathrm{H}_{2} \mathrm{O}, \mathrm{NaH}_{2} \mathrm{PO}_{4} \cdot 2 \mathrm{H}_{2} \mathrm{O}, \mathrm{MgSO}_{4} \cdot 7 \mathrm{H}_{2} \mathrm{O}$ and $\mathrm{Na}_{2} \mathrm{CO}_{3}$, for preparation of the $\mathrm{B}$-buffer, phosphate buffer and the quenching solutions, were purchased from Sigma-Aldrich (St. Louis, MO, USA). Tryptone was obtained from BD (Franklin Lakes,

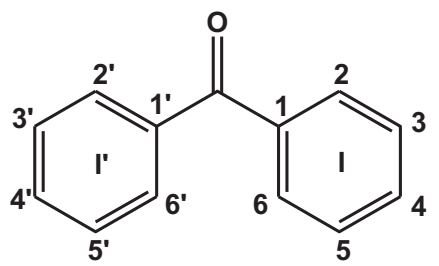

Fig. 1. The skeleton of benzophenone-type UV filters.
NJ, USA). Dimethylsulfoxide (DMSO), 4-(2-hydroxyehyl)-1-piperazineethane sulfonic acid (HEPES buffer), glucose, penicillin sodium, sodium dodecyl sulfate, $\beta$-sulfur ethanol, and $o$-Nitrophenyl- $\beta$-D-galactoside were purchased from Amresco (Solon, $\mathrm{OH}$, USA). The stock solutions of the BPs for the genotoxicity tests were prepared in DMSO.

\subsection{SOS/umu genotoxicity test}

A standard SOS/umu protocol recommended by ISO was performed to measure the genotoxicity of the BP-type UV-filters (ISO, 2000). The engineered bacterium, Salmonella thyphimurium TA1535 pSK1002, was obtained from Professor Yoshimitsu Oda (Japan) as a gift. The stock solutions of the chemicals were diluted for toxicity exposure, and the DMSO concentration was maintained at 1 percent in the exposure solution. In each test, 4-nitroquinoline-N-oxide (4-NQO) and 1percent DMSO were used as the positive control and the negative control, respectively. For each studied compound, an index of the lowest positive effect concentration (LPEC), defined as the concentration of the compound corresponding with the induction ratio (IR) of 2 on its dose-response curve, was determined. Generally, an induction ratio of 2 is considered to be indicative of a compound that has a positive genotoxicity effect (Oda et al., 1985; ISO, 2000). To facilitate analysis, the LPEC values for the studied compounds were converted to $p L E P C(-\lg L E P C)$ values. A high PLPEC value corresponds with a high genotoxicity for that compound. The genotoxicity tests were conducted in triplicate, and the results presented are the average values (Table 1 ).

\subsection{D-QSAR method}

The ADRIANA.Code program (Version 2.2.4) was applied to calculate the physicochemical parameters for the studied BPs. A total of 29 global molecular descriptors, 8 shape descriptors and 88 2D property-weighted autocorrelation (or topological) descriptors were obtained. These descriptors were selected as the independent variables, and the $P L P E C$ values were selected as the dependent variables. A stepwise multiple linear regression method was used to establish the QSAR equations.

\subsection{D-QSAR method}

A 3D-QSAR study for the genotoxicity of the BPs (described by the pLPEC values) was conducted using the comparative molecular similarity indices analysis (CoMSIA) (Klebe et al., 1994). The 3D structures of the molecules were constructed using the standard tools available in the Sybyl molecular modeling package (version 7.0). The partial atomic charges were calculated using the GasteigerHückel method. The energy minimization was performed using the Tripos force field with the Powell conjugate gradient algorithm and a convergence criterion of $0.005 \mathrm{kcal} / \mathrm{mol} \AA$. The template molecule chosen for this study was $2,4,4^{\prime}$-trihydroxy-BP (BP8), which is an asymmetric molecule with the highest genotoxicity (Xu et al., 2002). The database alignment method was applied to superimpose the 14 benzophenone compounds (Fig. 2).

For CoMSIA, the overlapped molecules were placed into a 3D cubic lattice with a $2 \AA$ grid. An $\mathrm{sp}^{3}$ hybridized carbon atom with a +1 charge served as the probe atom to generate steric and electrostatic field energy. A distance-dependent dielectric constant of 1.0 was used, and the default energy cutoff value of $30 \mathrm{kcal} /$ mol was applied. The regression analysis was performed using the partial least squares method. The cross-validation with the leave-one-out method was performed to yield the highest cross-validation correlation coefficient $\left(Q^{2}\right)$ and the optimum number of components $(N)$. Simultaneously, non-cross-validated analysis was performed, and the conventional multiple correlation coefficient $\left(R^{2}\right)$, standard error of estimate $(S E)$ and the Fisher test $(F)$ values were calculated.

\section{Results and discussion}

\subsection{Genotoxicity of the BP-type UV filters}

The genotoxicity results (described as $L P E C$ and $P L P E C$ ) for the 14 BP-type UV filters tested with the SOS/umu assay are listed in Table 1. The LPEC values for the 14 BPs ranged from 81.97 to $>1000 \mathrm{mg} / \mathrm{L}$. Among those 14 BPs, BP13 exhibited the lowest genotoxicity and showed no genotoxicity effect even at $1000 \mathrm{mg} / \mathrm{L}$. BP10 showed the strongest genotoxicity effect with an $L P E C$ value of $81.92 \mathrm{mg} / \mathrm{L}$. The studied compounds contained the same benzophenone skeleton, and differed only in the position, number and type of chemical substituents on the benzene rings. Therefore, it was necessary to investigate the contribution of the chemical substituents on the genotoxicity of the studied BP-type UV filters. 
Table 1

Fourteen BP-type UV filters and their genotoxicity data (LPEC and pLPEC).

\begin{tabular}{|c|c|c|c|c|c|c|}
\hline No & Compounds & Structure & MW & CAS no. & LPEC $(\mathrm{mg} / \mathrm{L})$ & pLPEC $(\mathrm{g} / \mathrm{L})$ \\
\hline BP1 & Benzophenone & & 182.22 & $119-61-9$ & 769.23 & 0.11 \\
\hline BP2 & 2-Hydroxyl benzophenone & & 198.22 & $117-99-7$ & 714.29 & 0.15 \\
\hline BP3 & 3-Hydroxyl benzophenone & & 198.22 & $13020-57-0$ & 625.00 & 0.20 \\
\hline BP4 & 4-Hydroxyl benzophenone & & 198.22 & $1137-42-4$ & 384.62 & 0.41 \\
\hline BP5 & 2,2'-Dihydroxyl benzophenone & & 214.22 & $835-11-0$ & 312.50 & 0.50 \\
\hline BP6 & 4,4'-Dihydroxyl benzophenone & & 214.22 & $611-99-4$ & 294.18 & 0.53 \\
\hline BP7 & 2,4-Dihydroxyl benzophenone & & 214.22 & $131-56-6$ & 102.04 & 0.99 \\
\hline BP8 & 2,4,4'-Trihydroxyl benzophenone & & 230.22 & $1470-79-7$ & 98.04 & 1.00 \\
\hline BP9 & 2,3,4-Trihydroxyl benzophenone & & 230.22 & $1143-72-2$ & 92.03 & 1.03 \\
\hline BP10 & $2,2^{\prime}, 4,4^{\prime}$-Tetrahydroxyl benzophenone & & 246.22 & $131-55-5$ & 81.97 & 1.09 \\
\hline BP11 & 2-Hydroxyl-4-methoxyl benzophenone & & 228.22 & $131-57-7$ & 357.14 & 0.45 \\
\hline BP12 & 2-Hydroxyl-4-octyloxyl benzophenone & & 326.44 & $1843-05-6$ & $>500$ & $>0.30$ \\
\hline BP13 & 2-Hydroxyl-4-methoxyl benzophenone-5-sulfonic acid & & 308.31 & $4065-45-6$ & $>1000$ & $>0$ \\
\hline BP14 & 2,2'-Dihydroxyl-4-methoxl benzophenone & & 244.25 & $131-53-3$ & 625.00 & 0.20 \\
\hline
\end{tabular}

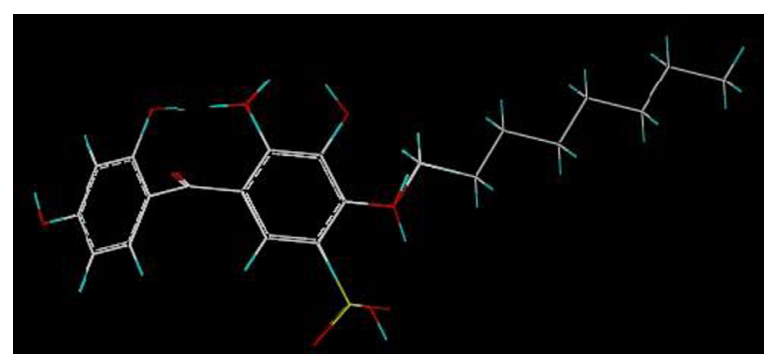

Fig. 2. Superposition of 14 benzophenones for CoMSIA studies.

\subsection{The effects of the hydroxy number on the genotoxicity}

Ten BPs (from BP1 to BP10) were selected to explore the effects that the number of hydroxy groups would have on the genotoxicity. Fig. 3 shows that the genotoxicity was enhanced as the number of hydroxy groups on the benzene rings increased. BP1, which does not contain a hydroxy group, exhibited the lowest genotoxicity, whereas BP10, with four hydroxy groups, exhibited the highest genotoxicity among the ten selected BPs. The genotoxicity of the BPs with three hydroxy groups (BP8 and BP9) was stronger than those with two hydroxy groups (BP5, BP6 and BP7) and much stronger than those with one hydroxy group (BP2, BP3 and BP4). This tendency is in accord with the reported results (Abramsson-Zetterberg and Svensson, 2011; Jeon et al., 2007). In addition, BP1 and 4-methoxy-BP were proven to be inactive in a micronucleus assay. BP8 had the most significant estrogenic and anti-androgenic activities, followed by BP6 and BP4, among the seventeen hydroxylated BPs (Suzuki et al., 2005). Another in vitro genotoxicity study of BP-type UV filters in L5178Y $\left(\mathrm{tk}^{+/-}\right)$mouse lymphoma cells showed that BP1, BP4, BP11 and BP7 did not 


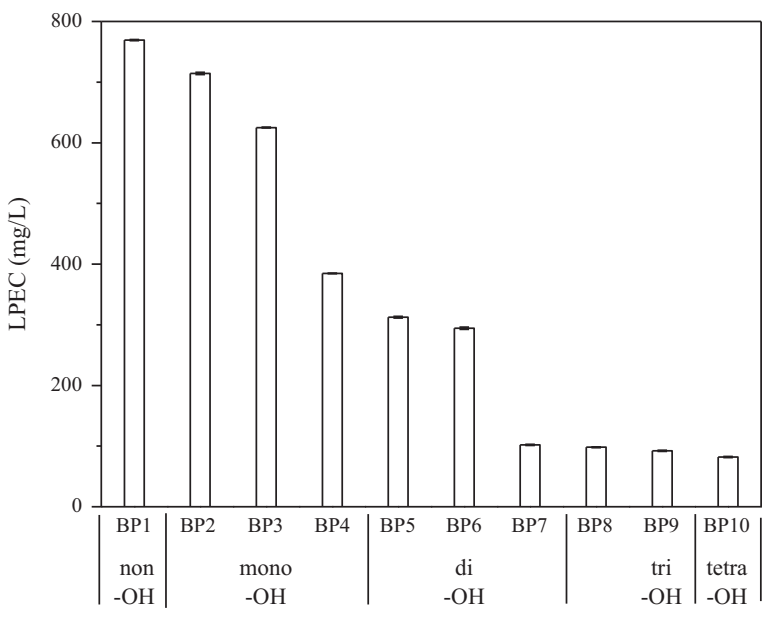

Fig. 3. The effects of hydroxy number on the genotoxicity.

induce significant mutation frequencies either in the presence or the absence of a metabolic activation system. BP14 exhibited positive results at $120.4 \mu \mathrm{g} / \mathrm{mL}$ in the absence of a metabolic activation system. BP9 revealed a significant increase in mutation frequencies in the range of $138.1-207.2 \mu \mathrm{g} / \mathrm{mL}$ and $118.3-354.8 \mu \mathrm{g} / \mathrm{mL}$ in the absence and presence, respectively of a metabolic activation system (Jeon et al., 2008).

\subsection{The effects of the hydroxy position on the genotoxicity}

In addition to the hydroxy number, the substitution position of the hydroxy group(s) on the benzene ring also influences the genotoxicity. For example, BP2, BP3 and BP4 each contain one hydroxy group, but the genotoxicity increased in the order of 2$<3$ - < 4-hydroxy-BP. Similarly, both 2,2'-dihydroxy-BP (BP5) and $4,4^{\prime}$-dihydroxy-BP (BP6) contain two hydroxy groups, but the genotoxicity of BP6 was stronger than that of BP5. Kawamura et al. (2003) measured the endocrine-disrupting effects of BP-type compounds and found that the endocrine-disrupting effects of the compounds with the hydroxy group located at the 4-position were the highest, followed by that located at the 3- and 2-position. This result suggested that the hydroxy group interacted with the carbonyl group, causing the BP skeleton to become more fixed and, thus, depending on the position of the hydroxy group, the volume of the hydrophobic moiety became a better fit for the endocrine receptor (Kawamura et al., 2003).

In addition, for the BP-type UV filters with the same number of hydroxy groups, the compounds with the hydroxy groups located on two benzene rings exhibit a weaker genotoxicity than those located on one benzene ring. For example, 2,3,4-trihydroxy-BP (BP9) had a stronger genotoxicity than 2,4,4'-trihydroxy-BP (BP8). Moreover, 2,4-dihydroxy-BP (BP7) exhibited a stronger genotoxicity than 2,2'-dihydroxy-BP (BP5) and 4,4'-dihydroxy-BP (BP6). This result did not agree with the structure-based rules for predicting estrogenicity, which may be due to the different toxicological interactions (Schultz et al., 2000).

\subsection{The effects of the alkoxy groups on the genotoxicity}

In addition to the hydroxy group, the impacts of other groups (e.g., methoxy, octyloxy, and the sulfonic acid group) on the genotoxicity was also explored. A comparison of BP2 with BP11 and BP12 revealed that the introduction of the methoxy or the octyoxy group at the 4-position increased the genotoxicity. In contrast, a comparison of BP7 with BP11 and BP12 revealed that the replacement of the hydroxy group by a methoxy or a octyloxy group caused a significant decrease in the genotoxicity. Similarly, a comparison of BP8 with BP14 revealed that the replacement of the hydroxy group by a methoxy group caused a decrease in genotoxicity. The effects of the alkoxy groups on the genotoxicity may be attributed to the damage to the hydrogen bond between the hydroxy group and the DNA bases when the hydroxy group was replaced by the alkoxy group. Moreover, the introduction of the octyloxy group would increase the molecular volume and block chemicals from entering into the cells (Schultz et al., 2000). In addition, a comparison of the genotoxicity of BP11 and BP13 revealed that the introduction of the sulfonic acid group in BP13 significantly decreased the genotoxicity. Because the sulfonic acid group greatly increases the hydrophilicity of BP13, a decreased concentration of the compound enters into the cells, therefore, decreasing the genotoxicity.

\subsection{D-QSAR analysis}

A two dimensional quantitative structure-activity relationship equation between genotoxicity ( $p L E P C)$ and the molecular structure descriptors was developed for the BP-type UV filters using a stepwise multiple linear regression analysis described by:

pLPEC $=0.035+0.285$ HDon_O

$N=14, R^{2}=0.714, R_{a d j}^{2}=0.686, S E=0.211, F=24.985, p<0.001$

where $N$ represents the number of samples, $R^{2}$ is the multiple correlation coefficient, $R_{a d j}^{2}$ is the multiple correlation coefficient adjusted by the free degree, $S E$ is the standard deviation, $F$ denotes the $F$-test value, and $p$ is the significance level of the equation.

As shown in Eq. (1), only one parameter, HDon_O (the number of hydrogen atom donors), was involvezd. The process of counting the number of hydrogen atom donors in a molecule complies with Lipinski's "Rule of 5" mnemonic (Lipinski et al., 1997). The total number of $\mathrm{O}-\mathrm{H}$ groups in a molecule reflects the hydrogen bond donor character of that molecule. The positive correlation of HDon_O to the genotoxicity ( $p L P E C$ ) implies that the genotoxicity is enhanced in relation to the increasing number of the hydroxy groups that are present in the molecule, which is in accord with the results discussed above. However, the specific contribution of the hydroxy groups on different positions of the benzophenone ring to the genotoxicity was not clear, and a 3D-QSAR study was necessary.

\section{6. $3 D-Q S A R$ analysis}

\subsubsection{CoMSIA model analysis}

A 3D-QSAR model between the genotoxicity (described by the PLPEC values) and the molecular field information of the BP-type UV filters was developed using the comparative molecular similarity indices analysis (CoMSIA). The statistical parameters of the CoMSIA model (CoMSIA-1) are given in Table 2. The contributions of the steric, electrostatic, acceptor, donor and hydrophobic fields were $2.1,13.7,19.7,58.4$ and 6.1 percent, respectively. Considering the small contribution of the steric and hydrophobic fields to the genotoxicity, an optimized CoMSIA model (CoMSIA-2) was established on the basis of the electrostatic, hydrogen bond acceptor and donor fields, in which the contributions were 14.7, 63.6, and 21.7 percent, respectively. Among these fields, the hydrogen bond donor field was the largest contributor in the CoMSIA-2 model, which is consistent with the results of the 2D-QSAR model, in which HDon_O is the only independent variable.

\subsubsection{Contour map analysis}

Fig. 4 shows the contour maps derived from the 3D-QSAR model CoMSIA-2. The template compound, BP8, is displayed in the 
Table 2

Statistical parameters of the CoMSIA models.

\begin{tabular}{|c|c|c|c|c|c|c|c|c|c|c|}
\hline & \multicolumn{5}{|c|}{ Statistic index } & \multicolumn{5}{|c|}{ Contribution (\%) } \\
\hline & $N$ & $Q^{2}$ & $R^{2}$ & SEE & $F$ & Steric & Electrostatic & Acceptor & Donor & Hydrophobic \\
\hline CoMSIA-1 & 2 & 0.613 & 0.911 & 0.129 & 45.923 & 2.1 & 13.7 & 19.7 & 58.4 & 6.1 \\
\hline CoMSIA-2 & 2 & 0.645 & 0.907 & 0.131 & 44.041 & _- & 14.7 & 21.7 & 63.6 & 1 \\
\hline
\end{tabular}

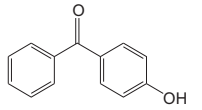

Fig. 4. The contour maps derived from the 3D-QSAR model CoMSIA-2. (a) electrostatic field, (b) hydrogen bond donor field, (c) hydrogen bond acceptor field.

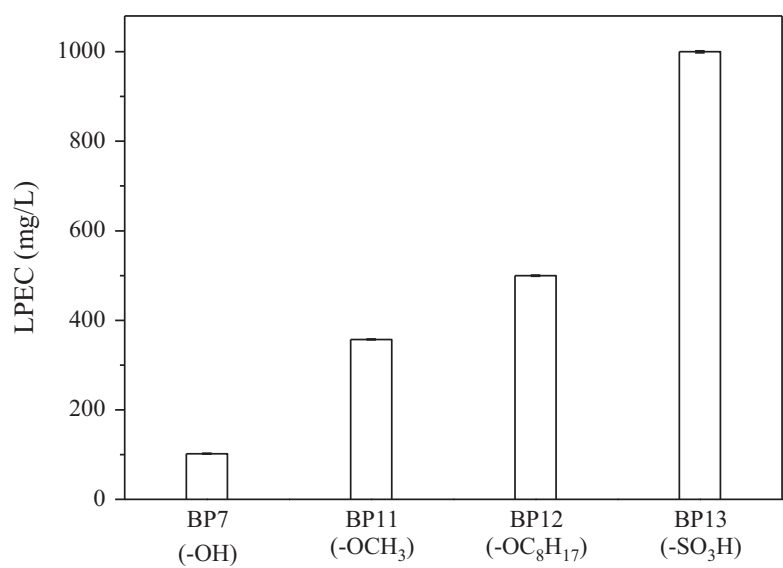

Fig. 5. The effects of substituent types on the genotoxicity.

background. The contribution of the substitution position and the substituent properties on the genotoxicity could be comprehensively revealed by analyzing the color, size and covering region of the contour maps.

The electrostatic contour map (Fig. 4a) reveals that the red and blue contours represent 80 and 20 percent of the contribution, respectively. The red contours indicate regions where negative charges increase toxicity, whereas the blue contours indicate regions where positive charges increase toxicity. A red contour surrounded the 4-position of benzene ring I, suggesting that the substituent with a negative charge on the 4-position would increase the genotoxicity. This result could be supported by comparing the genotoxicity of BP2 with BP10, BP11 and BP12. The presence of an oxygen-containing group, such as hydroxy, methoxy, and octyloxy, on the 4-position increased the genotoxicity due to the high electronegativity of the $\mathrm{O}$ atom. Moreover, the negative charge density on the three groups increased in the order of octyloxy $<$ methoxy $<$ hydroxy, which was in accord with the order of increasing genotoxicity (Fig. 5). In addition, two blue contours surrounded the 3-position and the substituent on the 5 -position, implying that an increase in the negative charge on these two positions would decrease genotoxicity. For example, the introduction of a $-\mathrm{SO}_{3} \mathrm{H}$ group (a strong electron-withdrawing group) onto the 5-position caused the corresponding compound, BP13, to exhibit the lowest genotoxicity among the studied BPs.

Due to the complicated substitution variations on the benzene rings of the benzophenone skeleton, the hydrogen-bond donor contour maps (Fig. 4b) could not provide clear information. Moreover, the major substituent on the benzophenone skeleton was the hydroxy group, which plays a dual role as a hydrogenbond donor and as an acceptor. Theoretically, the hydrogen-bond acceptor field and donor field would depict corresponding information on the contribution of the substituents. Therefore, the hydrogen-bond acceptor contour maps (Fig. 4c) were analyzed to elucidate the contribution of the hydrogen-bond interaction on the genotoxicity of the BP-type UV filters. For the hydrogen-bond acceptor contour maps, the amaranth contours indicated regions where the hydrogen-bond acceptor groups increased toxicity, whereas the red contours indicated regions where the groups decreased the toxicity. In Fig. 4c, two medium-sized amaranth blocks were close to the 2- and 4-positions on the benzene ring, suggesting that the introduction of a hydrogen-bond acceptor to the region would increase the genotoxicity. Therefore, it was expected that BP10 $(L P E C=81.97 \mathrm{mg} / \mathrm{L}), \mathrm{BP9}(L P E C=92.03 \mathrm{mg} / \mathrm{L})$ and BP7 $(L P E C=102.04 \mathrm{mg} / \mathrm{L}$ ) with 2,4-di-hydroxylated BPs would have a significantly higher genotoxicity than the other tested BPs. A medium-sized red contour surrounded the 5-position, implying that the sulfonic acid group, which acted as a hydrogen-bond acceptor, decreased the genotoxicity. Because the oxygen atoms in the $-\mathrm{SO}_{3} \mathrm{H}$ group are weaker hydrogen-bond acceptors than those in the hydroxy groups, BP11 $(L P E C=357.14 \mathrm{mg} / \mathrm{L})$ had a higher genotoxicity than BP13 $($ LPEC $>1000 \mathrm{mg} / \mathrm{L})$.

\section{Conclusion}

The genotoxicity of 14 benzophenones was measured using the SOS/umu assay, and a new index, the lowest positive effect concentration ( $L P E C$ ), was defined to describe the genotoxicity of the tested compounds. The LPEC values for the 14 BP-type UV filters ranged from 81.97 to $>1000 \mathrm{mg} / \mathrm{L}$; these values were dependent on the number, position and type of chemical substituents on the benzophenone skeleton. The genotoxicity trends that were revealed in this study are as follows: (i) The genotoxicity increased along with the number of hydroxyl substitutions. (ii) The genotoxicity increased in the order of the $2-<3-<4$-position for the benzophenones containing the same number of hydroxyls. (iii) The genotoxicity was reduced when the substituted hydroxyls were distributed between two benzene rings rather than on one benzene ring. These results were theoretically supported by the 2D- and 3D-QSAR studies. The physicochemical parameter HDon_O had a positive contribution to the genotoxicity in the 2D-QSAR model. The hydrogen-bond acceptor field information in the CoMSIA study implied that the introduction of an acceptor group containing high electronegativity atoms to the 2- and 4-positions would increase genotoxicity. The present study provided direct toxicity data for the BP-type UV filters, which is beneficial for the scientific evaluation of the environmental fate and potential hazard of UV filters.

\section{Acknowledgments}

This work was supported by the National Natural Science Foundation of China (Projects 20877090, 50938004 and 21077123) and the MOST (Grant 2009CB421605). 


\section{References}

Abramsson-Zetterberg, L., Svensson, K., 2011. 4-Methylbenzophenone and benzophenone are inactive in the micronucleus assay. Toxicol. Lett. 201, 235-239.

Balmer, M.E., Buser, H.R., Muller, M.D., Poiger, T., 2005. Occurrence of some organic UV filters in wastewater, in surface waters, and in fish from Swiss lakes. Environ. Sci. Technol. 39 (4), 953-962.

Burdock, G.A., Pence, D.H., Ford, R.A., 1991. Safety evaluation of benzophenone. Food Chem. Toxicol. 11, 741-750.

Dutta, K., Das, M., Rahman, T., 1993. Toxicological impacts of benzophenone on the liver of guinea pig. Bull. Environ. Contam. Toxicol. 50, 282-285.

Fent, K., Kunz, P.Y., Zenker, A., 2010. A tentative environmental risk assessment of the UV-filters3-(4-methylbenzylidene-camphor), 2-ehyl-hexyl-4-trimethoxycinnamate, benzophenone -3 , benzophenone-4 and 3-benzylidenne camphor. Mar. Environ. Res. 69 (Suppl. 1), S4-S6.

ISO (International Standard Organisation), 2000. Water quality-determination of the genotoxicity of water and waste water using the umu-test (1st ed.) ISO 13829, Geneva, pp. 1-18.

Jeon, H., Chung, Y., Ryu, J., 2006. Simultaneous determination of benzophenonetype UV fliters in water and soil by gas chromatography-mass spectrometry. J. Chromatogr. A 1131, 192-202.

Jeon, H.K., Sarma, S.N., Kim, Y.J., Ryu, J.C., 2007. Forward gene mutation assay of seven benzophenone-type UV filters using L5178Y mouse lymphoma cell. Mol. Cell. Toxicol. 3 (1), 23-30.

Jeon, H.K., Sarma, S.N., Kim, Y.J., Ryu, J.C., 2008. Toxicokinetics and metabolisms of benzophenone-type UV filters in rats. Toxicol. 248, 89-95.

Kawamura, Y., Ogawa, Y., Nishimura, T., Kikuchi, Y., Nishikawa, J., Nishihara, T. Tanamoto, K., 2003. Estrogenic activities of UV stabilizers used in food contact plastics and benzophenone derivatives tested by the yeast two-hybrid assay. J. Health Sci. 49 (3), 205-212.

Klebe, G., Abraham, U., Mietzner, T., 1994. Molecular similarity indices in a comparative analysis (CoMSIA) of drug molecules to correlate and predict their biological activity. J. Med. Chem. 37 (24), 4130-4146.

Kunisue, T., Chen, Z., Buck Louis, G.M., Sundaram, R., Hediger, M.L., Sun, L., Kannan, K., 2012. Urinary concentrations of benzophenone-type UV filters in U.S. women and their association with endometriosis. Environ. Sci. Technol. 46, 4624-4632.

Kunz, P.Y., Fent, K., 2006. Estrogenic activity of UV filter mixtures. Toxicol. Appl. Pharmacol. 217, 86-99.

Kunz, P.Y., Galicia, H.F., Fent, K., 2006. Comparision of in vitro and in vivo estrogenic activity of UV fliters in fish. Toxicol. Sci. 90, 349-361.

Kupper, T., Plagellat, C, Braendli, R.C., Grandjean, D., Tarradellas, J., 2006. Fate and removal of polycyclic musks, UV filters and biocides during wastewater treatment. Water Res. 40 (14), 2603-2612.

Kusk, K.O., Avdolli, M., Wollenberger, L., 2011. Effect of 2,4-dihydroxybenzophenone (BP-1) on early life-stage development of the marine copepod Acartia tonsa at different temperature and salinities. Envion. Toxicol. Chem. 30 (4), 959-966.

Li, W.H., Ma, Y.M., Guo, C.S., Hu, W., Liu, K.M., Wang, Y.Q., Zhu, T., 2007. Occurrence and behavior of four of the most used sunscreen UV fliters in a wastewater reclamation plant. Water Res. 41 (15), 3506-3512.

Lipinski, C.A., Lombardo, F., Dominy, B.W., Feeney, P.J., 1997. Experimental and computational approaches to estimate solubility and permeability in drug discovery and development settings. Adv. Drug Delivery Rev. 23, 3-25.

Liu, Y.S., Ying, G.G., Shareef, A., Kookana, R.S., 2012. Occurrence and removal of benzotriazoles and ultraviolet filters in a municipal wastewater treatment plant. Environ. Pollut. 165 (6), 225-232.

Loraine, G.A., Pettigrove, M.E., 2006. Seasonal variations in concentrations of pharmaceuticals and personal care products in drinking water and reclaimed wastewater in southern California. Environ. Sci. Technol. 40 (3), 687-695.

Matsumoto, K., Akao, Y., Kobayashi, E., Ito, T., Ohguchi, K., Tanaka, T., Iinuma, M., Nozawa, Y., 2003. Cytotoxic benzophenone derivatives from Garcinia species display a strong apoptosis-inducing effect against human leukemia cell lines. Biol. Pharm. Bull. 26, 569-571.

Nakajima, D., Asada, S., Kageyama, S., Yamamoto, T., Kuramochi, H., Tanaka, N., Takeda, K., Goto, S., 2006. Activity related to carcinogenicity of plastic additives in the benzophenone group. J. UOEH 28, 143-156.

Ministry of Environment (MOE), 2000. MOE's perspectives on endocrine disrupting effects of substances-Strategic Programs on Environmental Endocrine Disruptors (SPEED'98). 〈http://www.env.go.jp/chemi/end/endindex.html. Japan〉.
Negreira, N., Rodriguez, I., Rubi, E., Cela, R., 2010. Dispersive liquid-liquid microextraction followed by gas chromatography-mass spectrometry for the rapid and sensitive determination of UV filters in environmental water samples. Anal. Bioanal. Chem. 398 (2), 995-1004.

Negreira, N., Rodriguez, I., Rubi, E., Cela, R., 2011. Optimization of pressurized liquid extraction and purification conditions for gas chromatography-mass spectrometry determination of UV fliters in sludge. J. Chromatogr. A 1218 (31), 211-217.

Oda, Y., Nakamura, S., Oki, I., Kato, T., Shinagawa, H., 1985. Evaluation of thenewsystem (umu-test) for the detection of environmental mutagens and carcinogens. Mutat. Res. 147, 219-229.

Okereke, C.S., Barat, S.A., Abdel-Rahman, M.S., 1995. Safety evaluation of benzophenone-3 after dermal administration in rats. Toxicol. Lett. 80, 61-67.

Poiger, T., Buser, H.R., Balmer, M.E., Bergqvist, P.A., Müller, M.D., 2004. Occurrence of UV filter compounds from sunscreens in surface waters: regional mass balance in two Swiss lakes. Chemosphere 55 (7), 63-951.

Rodil, R., Moeder, M., 2008. Development of a method for the determination of UV fliters in water samples using stir bar sorptive extraction and thermal desorption-gas chromatography-mass spectrometry. J. Chromatogr. A 1179 (2), 81-88.

Roelandts, R., Vanhee, J., Bonamie, A., Kerkhofs, L., Degreef, H., 1983. A survey of ultraviolet absorbers in commercially available sun products. Int. J. Dermatol. 22 (40), 247-255

Schauder, S., Ippen, H., 1997. Contact and photocontact sensitivity to sunscreens. Review of a 15 year experience and of the literature. Contact Derm. 37 221-232.

Schlumpf, M., Schmid, P., Durrer, S., Conscience, M., Maerkel, K., Henseler, M. Gruetter, M., Herzog, I., Reolon, S., Ceccatelli, R., Faass, O., Stutz, E., Jarry, H., Wuttke, W., Lichtensteiger, W., 2004. Endocrine activity and developmental toxicity of cosmetic UV filters-an update. Toxicology 205, 113-122.

Schultz, T.W., Seward, J.R., Sinks, G.D., 2000. Estrogenicity of benzophenones evaluated with a recombinant yeast assay: comparison of experimental and rules-based predicted activity. Environ. Toxicol. Chem. 19 (2), 301-304.

Sieratowicz, A., Kaiser, D., Behr, M., Oetken, M., Oehlmann, J., 2011. Acute and chronic toxicity of four frequently used UV filter substances for Desmodesmus subspicatus and Daphnia magna. J. Environ. Sci. Health Part A 46, 1311-1319.

Suzuki, T., Kitamura, S., Khota, R., Sugihara, K., Fujimoto, N., Ohta, S., 2005 Estrogenic and antiandrogenic activities of 17 benzophenone derivatives used as UV stabilizers and sunscreens. Toxicol. Appl. Pharmacol. 203, 9-17.

Tomson, M.B., Dauchy, J., Hutchins, S., Curran, C., Cook, C.J., Ward, C.H., 1981. Groundwater contamination by trace level organics from a rapid infiltration site. Water Res. 15, 1109-1116.

Thomas, P., Hans-Rudolf, B., Marianne, E.B., Per-Anders, B.D., Markus, M., 2004 Occurrence of UV filter compounds from sunscreens in surface waters: regional mass balance in two Swiss lakes. Chemosphere 55, 951-963.

Vosough, M., Mojdehi, N.R., 2011. Fast liquid chromatography-diode array detection assisted by chemometrics for quantification of seven ultraviolet filter in effluent wastewater. Talanta 85 (4), 2175-2181.

Wang, L., Asimakopoulos, A.G., Moon, H.-B., Nakata, H., Kannan, K., 2013. Benzotriazole, benzothiazole and benzophenone compounds in indoor dust from the United States and east Asia. Environ. Sci. Technol. 47, 4752-4759.

Wei, D.B., Zhang, A.Q., Wei, Z.B., Han, S.K., Wang, L.S., 2002. A case study of logistic QSAR modeling methods and robustness tests. Ecotoxicol. Environ. Saf. 52 (2), 143-149.

Weisbrod, C.J., Kunz, P.Y., Zenker, A.K., Fent, K., 2007. Effects of the UV filter benzophenone-2 on reproduction in fish. Toxicol. Appl. Pharmacol. 225 255-266.

West, C.E., Rowland, S.J., 2012. Aqueous phototransformation of diazepam and related human metabolites under simulated sunlight. Environ. Sci. Technol. 46 4749-4756.

Xu, M., Zhang, A.Q., Han, S.K., Wang, L.S., 2002. Studies of 3D-quantitative structure-activity relationships on a set of nitroaromatic compounds: CoMFA advanced CoMFA and CoMSIA. Chemosphere 48, 707-715.

Zhang, Z., Ren, N., Li, Y.F., Kunisue, T., Gao, D., Kannan, K., 2011. Determination of benzotriazole and benzophenone UV fliters in sediment and sewage sludge. Environ. Sci. Technol. 45 (9), 3909-3916.

Zucchi, S., Bluthgen, N., Ieronimo, A, 2011. The UV-absorder benzophenone-4 alters transcripts of genes involved in hormonal pathways in zebrafish(Danio rerio) eleuthero-embryos and adult males. Toxicol. Appl. Pharmacol. 250 (2), 137-146. 